\title{
Disección coronaria traumática: un partido de infarto
}

\section{Traumatic coronary dissection: A heart attack game}

Pilar Alcantud-Lozano ${ }^{1}$, Juan J. Portero-Portaz²*, Vicente Ferrer-Bleda², Sara Díaz-Lancha², Juan G. Córdoba-Soriano y Jesús M. Jiménez-Mazuecos²

${ }^{1}$ Servicio de Urgencias, Hospital de Hellín, Hellín; ${ }^{2}$ Servicio de Cardiología, Complejo Hospitalario Universitario de Albacete, Albacete, España
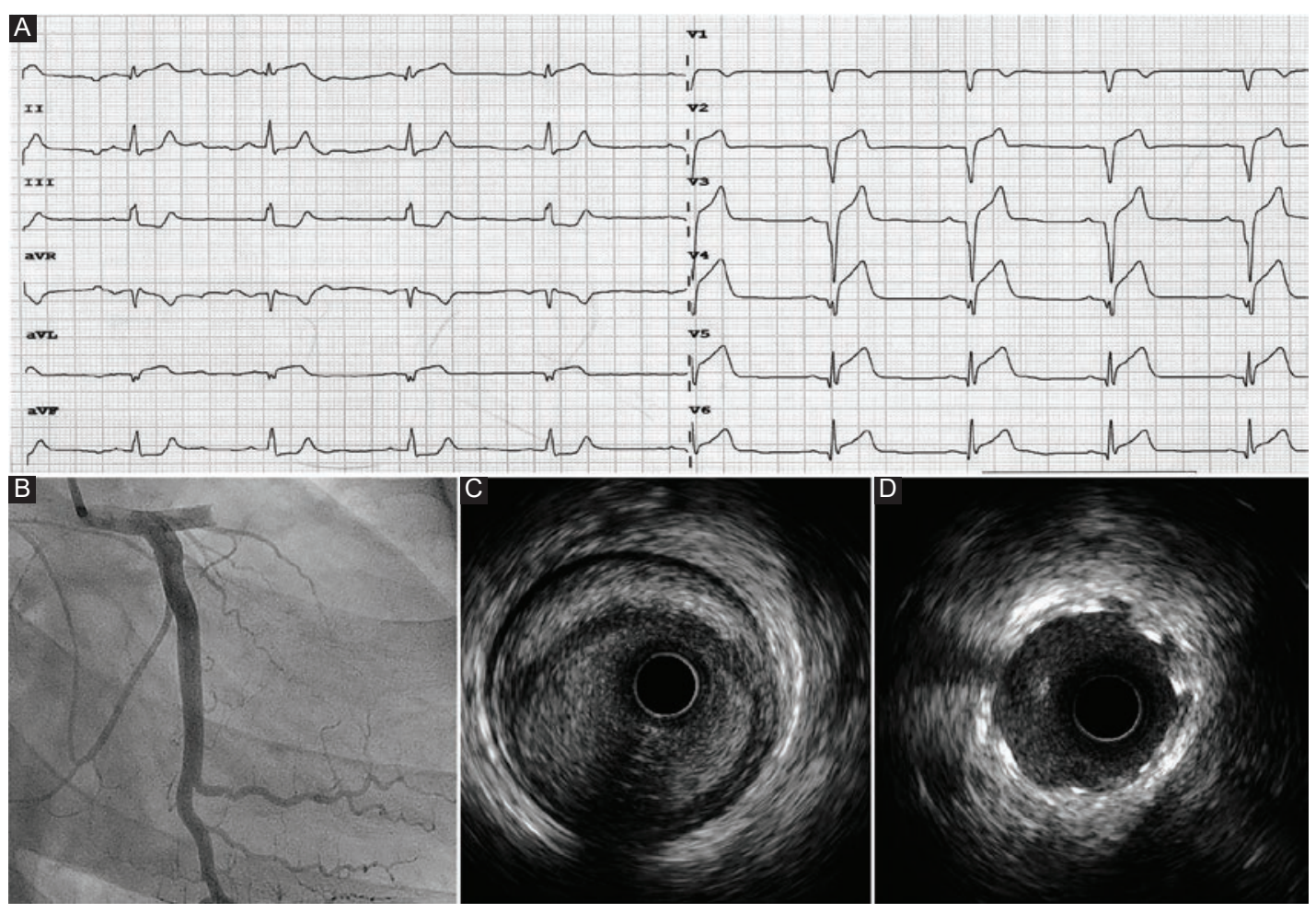

Figura 1. A: electrocardiograma que registra infarto con elevación del segmento ST de localización anterolateral. B: angiografía de la arteria coronaria izquierda (Oblicua anterior derecha $[0 A D]+20^{\circ}$, Caudo [CAU] $+20^{\circ}$ ) con oclusión aguda proximal de DA. C: USIV preangioplastia sobre DA con imagen de disección coronaria oclusiva y hematoma intramural (imagen en menisco o semilunar). D: USIV postangioplastia con implante de stent recubierto; se observan aposición y expansión correctas (struts hiperrefringentes).

\section{Correspondencia:}

*Juan J. Portero-Portaz

E-mail: Juanjose.porteroportaz@gmail.com
Fecha de recepción: 30-08-2019

Fecha de aceptación: 10-01-2020 DOI: 10.24875/ACM.20000336
Disponible en internet: 06-02-2020 Arch Cardiol Mex. 2020;90(2):222-223

www.archivoscardiologia.com

1405-9940/@ 2020 Instituto Nacional de Cardiología Ignacio Chávez. Publicado por Permanyer. Este es un artículo open access bajo la licencia CC BY-NC-ND (http://creativecommons.org/licenses/by-nc-nd/4.0/). 
Varón de 34 años sin factores de riesgo cardiovascular y jugador semiprofesional de rugbi. Consulta por dolor torácico luego de un traumatismo torácico (TT) durante un partido; presenta mal estado general, hematoma y dolor a la palpación esternocostal. Ante mala evolución con analgesia se realiza ECG (Fig. 1A); en virtud de los hallazgos se solicitó coronariografía urgente que delineó disección oclusiva de descendente anterior (DA) (Fig. 1B), con imagen de hematoma intramural en el ultrasonido intravascular (USIV; Fig. 1C); el paciente se trató con implante de stents farmacoactivos (Fig. 1D).

Los TT cerrados de alta energía, incluidos los observados en deportes de contacto ${ }^{1}$, pueden producir lesiones graves en órganos internos. Aunque infrecuente, la disección coronaria traumática es difícil de diagnosticar cuando la forma de presentación clínica, la epidemiología y las características del paciente no revelan un síndrome coronario. La fisiopatología se desconoce en buena medida, pero, al igual que otros grandes vasos, las fuerzas de tracción-distracción parecen ser las causas. No existen claros factores predisponentes; según algunos autores, las alteraciones del colágeno y la aterosclerosis no significativa pueden modificar la estructura arterial y hacerla vulnerable.

La revascularización percutánea resulta de elección en casos de oclusión o estenosis grave. En general no se considera la trombólisis debido a la diferente etiopatogenia. Se han descrito casos de tratamiento conservador con buenos resultados en lesiones no obstructivas, incluida la revascularización quirúrgica. La imagen intracoronaria, aunque controvertida, completa el diagnóstico y ayuda a seleccionar el tratamiento ${ }^{2}$.

Si bien existen centros con protocolos establecidos, no es aventurado afirmar que ante todo TT de alta energía, o en aquéllos más ligeros, pero con dolor incoercible o afectación clínica discordante con la exploración, son obligados la seriación de marcadores de necrosis y el ECG.

\section{Financiamiento}

Este trabajo no ha requerido financiamiento alguno para su elaboración.

\section{Conflicto de intereses}

No existe ningún conflicto de intereses en los autores.

\section{Responsabilidades éticas}

Protección de personas y animales. Los autores declaran que para esta investigación no se han realizado experimentos en seres humanos ni en animales.

Confidencialidad de los datos. Los autores declaran que han seguido los protocolos de su centro de trabajo sobre la publicación de datos de pacientes.

Derecho a la privacidad y consentimiento informado. Los autores han obtenido el consentimiento informado de los pacientes o sujetos referidos en el artículo. Este documento obra en poder del autor de correspondencia.

\section{Bibliografía}

1. Hazeleger R, van der Wieken R, Slagboom T, Landsaat P. Coronary dissection and occlusion due to sports injury. Circulation. 2001; 103(8):1174-1175.

2. Moreno R, Pérez del Todo J, Nieto M, Alba F, Alfonso F, García-Rubira JC, et al. Primary stenting in acute myocardial infarction secondary to right coronary artery dissection following blunt chest trauma. Usefulness of intracoronary ultrasound. Int J Cardiol. 2005;103(2):209-211. 